\title{
Study on Dijkstra Path Planning of Sensor Networks Based on Optimized Ant Colony Algorithm
}

\author{
Sun Jing \\ School of Mechanical and Materials Engineering, Xi'an University,710065
}

Keywords: Ant colony algorithm; Wireless sensor network; Robot; Path planning; Dijkstra

\begin{abstract}
With the continuous development of various sensor network positioning technologies, the accuracy of robot path planning based on wireless sensor networks has been continuously improved. Therefore, a Dijkstra path planning method for sensor networks based on optimized ant colony algorithm is proposed. Firstly, the CC2430 sensor network communication node module is designed based on the IEEE802.15.4 standard, and the CC2430 packet header format is modified to realize remote communication between nodes. Finally, the ant colony optimization is used to improve the dijkstra shortest path algorithm. The experimental results show that compared with the traditional algorithm, the proposed algorithm has a lower path planning length, can effectively complete the path planning optimization, and can meet the requirements of a variety of path navigation.
\end{abstract}

\section{Introduction}

With the continuous development of computer control technology, various path planning technologies have received more and more attention. Robot path planning applications generally need to solve three major problems [1], including: 1) accurate positioning, 2) environmental data collection [3], 3) efficient path planning [4]. Among them, perceptual environment information and path planning navigation is a problem that robot path planning needs to focus on, which directly affects the final effect of robot path planning [5]. At present, researchers have put forward many researches on path planning of wireless sensor networks [6]. In [7], a robot path planning system based on wireless sensor network is proposed, which can fuse multi-sensor information to improve the accuracy of the control system. Literature [8] proposed a wireless sensor network data acquisition algorithm suitable for robot path planning, which can reduce communication delay and work node sensor energy consumption. Literature [9] proposed a cortical excitation sensor fusion network for robot path planning motion estimation.

Based on the above research, this paper designs a Dijkstra path planning method based on ant colony algorithm, and proposes an optimized path planning algorithm. Firstly, the sensor network communication node module is designed based on IEEE802.15.4 protocol. Then use CC2430 hardware node to build a corresponding wireless sensor network, and modify the header format to achieve remote communication of the machine node. Finally, the ant colony optimization is used to improve the dijkstra shortest path algorithm. The experimental results show that compared with the traditional algorithm, the path planning algorithm proposed in this paper has a higher accuracy and work efficiency.

\section{Sensor Network Communication Node Module Design}

In this paper, the sensor network node adopts CC2430 wireless transceiver hardware module, and its communication standard is $2.4 \mathrm{GHz}$ IEEE802.15.4 protocol. The module has a wide range of application and low cost, and has a rich peripheral interface circuit. Therefore, CC2430 wireless transceiver modules are often used to build wireless sensor networks. CC2430 wireless transceiver module uses SPI communication bus and microprocessor chip (AT89C52 microcontroller) to connect, can achieve full duplex, synchronous data transmission. The connection mode of CC2430 wireless transceiver module and AT89C52 microcontroller is consistent with literature [7]. After the sensing network node CC2430 establishes a connection with the mobile robot, it needs to feed back 
the information collected by the mobile robot to the central node in real time. Therefore, the communication between sensor network node CC2430 and the central node is very important. In order to discriminate between the format of the transmitted data signal and the data signal received by the central node, the header content of the CC2430 is modified as follows:

struct CC2430_header\{

uint8 Application_ID; uint8 P_Length; uint8 HOP; uint8 Message_T; uint8 Message_G;

uint8 Acknowledgment_Numbe; uint16 CC2430_Desti_PANID; uint16 CC2430_cID; uint16 CC2430_len, uint8 CC2430_radius;

uint16 CC2430_P=Acknowledgment_Numbe +1; uint16 FCF; \}

\section{Dijkstra Path Planning and Design Based on Ant Colony Algorithm}

\section{Dijkstra Shortest Path Algorithm}

The Dijkstra algorithm is a simple optimal path finding algorithm that can be solved in ascending order of path length. Assuming that the weighted directed graph is $G$ and the source point is $S$, the shortest path from the remaining vertices of $S$ to $S$ is required. Assume that $V$ is a set of vertices in $\mathrm{G}, \mathrm{P}$ is the set of vertices that have been found (the shortest path to $\mathrm{S}$ ), and $\mathrm{Q}$ is the set of vertices that have not yet been found (the shortest path to $S$ ). Each component $D[i]$ in the vector $D$ represents the length of the shortest path from the source point $S$ to the vertex vi.

The program flow of using Dijkstra algorithm to complete the initial path planning is shown in Figure 5. After setting the source point $S$ and the end point $T$, this paper first finds the shortest path from $\mathrm{S}$ to $\mathrm{T}$ in the map through the Dijkstra algorithm. This article uses this path as the initial value of the ant colony algorithm for optimization.

\section{Ant Colony Optimization Algorithm}

Assume that the initial sub-optimal path based on Dijkstra's algorithm passes through points S, $\mathrm{P} 1, \mathrm{P} 2$, and so on. If any two targets on the connection path are Pi1 and Pi2, then the connection path can be represented by formula (1):

$$
P_{i}\left(h_{i}\right)=P_{i 1}+\left(P_{i 2}-P_{i 1}\right) \times h_{i} h_{i} \in[0,1], i=1,2, \ldots
$$

So the path length from source $S$ to end $\mathrm{T}$ can be expressed as:

$$
L=S, P_{1}\left(h_{1}\right)+\sum_{i=1}^{d-1} \text { length }\left\{P_{i}\left(h_{i}\right), P_{i+1}\left(h_{i+1}\right)+P_{d}\left(h_{d}\right) \ldots .\right\}
$$

Different combinations $h_{1}, h_{2}, \ldots, h_{d}$ can represent all possible and reasonable paths. Therefore, the problem of finding the best path through the ant colony algorithm is to find a suitable value $h_{1}, h_{2}, \ldots, h_{d}$. Let ant nest be the number R of ants, and the set of elements that need to be optimized is $\mathrm{D}$, which $D_{\phi_{i}}$ represents its third element ${ }^{(1 \leq i \leq n)}$. The variable that needs to be optimized in this article is $h_{1}, h_{2}, \ldots, h_{d}$, the quantity is $\mathrm{n}$. Assuming that these elements ${ }^{{ }_{i}}$ have $\mathrm{K}$ values, they are the pheromone $\zeta_{j}\left(D_{\varphi_{i}}\right)(0)$ of the first element under initial conditions [10].

According to formula (3), the first ant calculates [11] its parameter to distinguish the probability of each possible value.

$$
k\left(\zeta_{j}^{t}\left(D_{\varphi_{i}}\right)\right)=\frac{\zeta_{j}\left(D_{\varphi_{i}}\right)}{\sum_{i=1}^{n} \zeta_{j}\left(D_{\varphi_{i}}\right)}
$$

Then select elements from the set of large probabilities and adjust them according to equation (4). 


$$
\zeta_{j}\left(D_{\varphi_{i}}\right)(t+\Delta)=\zeta_{j}\left(D_{\varphi_{i}}\right)(t)+\Delta \zeta_{j}\left(D_{\varphi_{i}}\right)
$$

Among them, the increment of information on the element indicates the sum of the pheromone left by all the ants passing through this element. The calculation method is as follows:

$$
\Delta \zeta_{j}\left(D_{\varphi_{i}}\right)=\sum_{k}^{R} \Delta \zeta_{j}^{k}\left(D_{\varphi_{i}}\right)
$$

Repeat the above process until the maximum number of iterations is allowed, or all ants get a unique element, which is optimized.

\section{Learning and Training Steps}

The ant colony optimization learning and training steps are as follows:

Step 1: Select the initial sample and determine the parameters;

Step 2: For all training sample sets, all transitions are triggered in turn according to formula (2);

Step 3: Calculate the error cost function according to the following formula (6);

$$
E=2^{-1} \sum_{l=1}^{r} \sum_{i=1}^{n}\left(M_{l}\left(p_{i}\right)-M_{l}^{E}\left(p_{i}\right)\right)^{2}
$$

Among them, the number $\mathrm{n}$ of output libraries, the number $\mathrm{r}$ of sample sets, the actual token value $M_{l}\left(P_{i}\right)$ of the output library, and the expected token value $p_{i}$ of the output library. If the result $M_{l}^{E}\left(p_{i}\right)$ is less than the expected value ${ }^{p_{i}}$, stop; otherwise, proceed to the next step.

Step 4: Use the proposed ant colony optimization algorithm to adjust the value ${ }^{h_{1}, h_{2}, \ldots, h_{d}}$;

Step 5: Return to step 2 until the error is satisfied.

\section{Simulation Results and Analysis}

In order to analyze and verify the Dijkstra path planning method of the optimization ant colony algorithm sensing network presented in this paper, a specific experiment was carried out. The experimental hardware environment is: Intel Core i7 $2.2 \mathrm{GHz}$ processor, $8 \mathrm{G}$ memory. Experimental software environment: Windows 7 operating system, Matlab7.0 simulation software. The optimal path after ant colony optimization is shown in Figure 1.

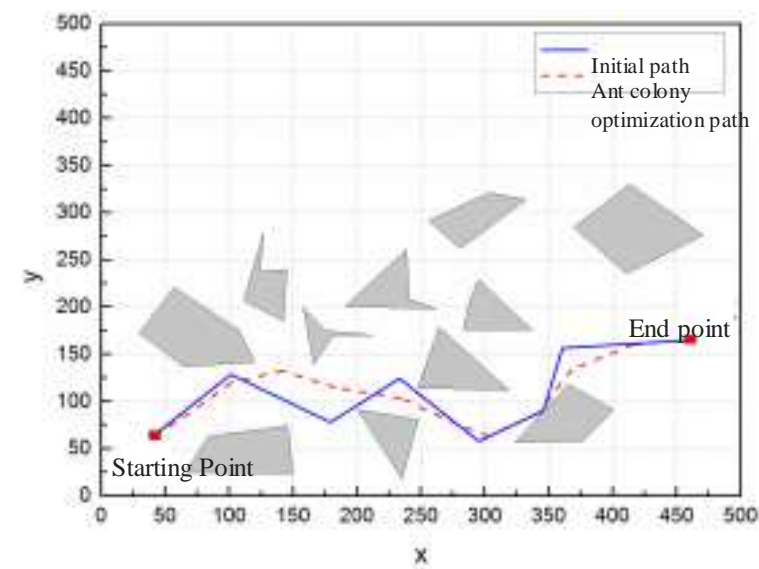

Figure 1. Ant Colony Algorithm Path Planning Results

In order to compare and analyze the proposed algorithm, under the condition of experimental

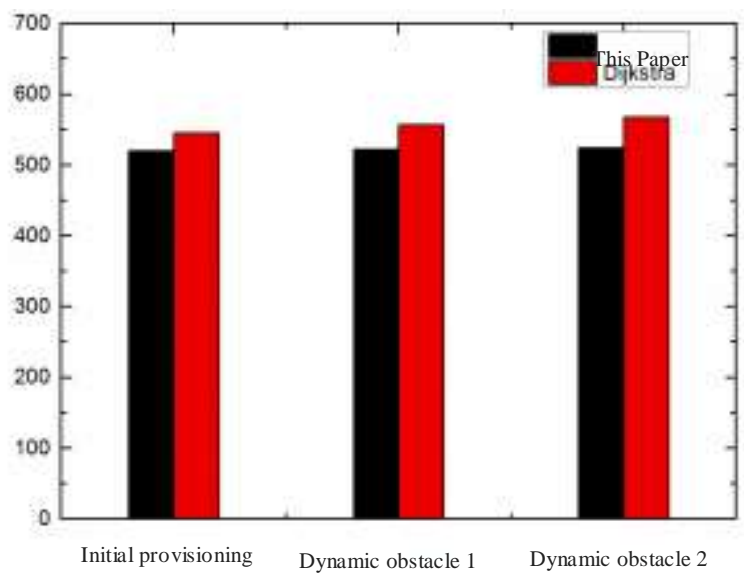

Figure 2. Path planning time parameters, this paper compared the algorithm with the traditional Dijkstra algorithm. The two different algorithms get the dynamic optimal planning length as shown in Fig. 2. From Figure 2, we can see that compared with the traditional Dijkstra algorithm, the length of the planned path obtained by the algorithm in all cases is the smallest. 


\section{Conclusion}

This paper designs the sensor network communication node module, and proposes a Dijkstra path planning method based on the optimized ant colony algorithm. Firstly, a corresponding wireless sensor network is constructed using CC2430 hardware nodes so as to realize remote communication between sensor network nodes. Then, the ant colony optimization was used to improve the dijkstra shortest path algorithm. The following conclusions are drawn: 1) Compared with other methods, the proposed method has smaller planning path length and higher robustness; 2) The proposed algorithm has the best overall performance. The designed path planning algorithm satisfies the requirements of path navigation.

\section{Problems and Improvements in Teaching}

The current era is an age of informatization. In the fields of industry and science and technology, information is mainly obtained through measurement. Modern industrial production is inseparable from accurate measurement data. Material flow and energy flow must be guided by measurement data to achieve orderly movement. Measurement and control technology has become an essential basic technology in modern production. In order to meet the needs of this development, our country's higher education system must cultivate professional talents with solid basic knowledge and good innovation.

The measurement and control technology and instrumentation specialty is multidisciplinary, involving a wide range of knowledge. It mainly involves measurement and control technology, instrumentation technology, computer technology, information technology and other fields. China's measurement and control technology and instrumentation specialty is an emerging specialty that has been broadened and merged on the basis of traditional precision instrument manufacturing, metrology and testing technologies, and optical instruments. The students of measurement and control technology and instrumentation mainly focus on the fields of measurement and control technology, intelligent instrument design, sensor design, information technology, and computer application technology. With the development of China's economy and society and the continuous improvement of industrialization, the measurement and control technology and instrumentation specialty has developed into a major science and technology major supported by China. It shoulders the heavy responsibility of cultivating professional information technology talents for instrumentation in China's modernization construction. .

Some people say that information technology is the key to the new technology revolution. The measurement and control technology and instrument specialty are the foundation and core of the key. As the main position for talent cultivation and output in China, colleges and universities shoulder the important task of cultivating and exporting talents for modernization. Strengthening the construction of measurement and control technology and instrument specialty in colleges and universities is one of the key areas for the deepening of teaching reform in universities.

First of all, we should base our teaching reform on measurement and control technology and instrumental expertise as a whole and intensify the establishment of a curriculum system. At present, in the reform of education and teaching in China, some schools adopt the method of repeatedly deleting the specific knowledge points by deleting the contents of teaching from simple to simple, although in the short term, relatively rapid teaching reform effects can be obtained, but from a whole point of view, a single deletion. The means lacks overall coordination and systemic perfection in the education and teaching system, and cannot fundamentally solve the contradiction between education and teaching effects and social needs. Therefore, the reform of the measurement and control technology and instrumental specialty should be considered and implemented from the overall perspective, from a system perspective, and proceed from the overall perspective to local optimization. 
Secondly, the experimental teaching of measurement and control technology and instrumentation is an important part of the teaching content of the measurement and control technology and instrument specialty, and it is the main way to cultivate the application-oriented talents for measurement and control technology and instrumentation in China. Traditional Chinese measurement and control technology and instrumentation are often bundled with theoretical teaching in experimental teaching. The experimental content is not only too simple, but there are still a lot of experimental repetitions in the experimental design, resulting in students not much interest in the experimental class, can not help students absorb and master The experimental purpose of knowledge of measurement and control technology and instrumental professional concepts. Therefore, in order to solve the main problems existing in the experiment teaching of China's measurement and control technology and instrumental specialty, colleges and universities should pay attention to the essence of experiment course in teaching reform. In the design of the experimental class, we should scientifically plan the content of the experimental class based on the actual needs of an outstanding professional talent for measurement and control technology and instrumentation in our society. In the experiment, we strive to allow students to reach through the perceptual thinking and threading of theoretical knowledge. Fully absorb and master the professional knowledge of measurement and control technology and instrumentation to meet students' needs for practical work experience in the future and improve their practical ability. We should establish a new experimental class assessment system based on students. The traditional evaluation system for measurement and control technology and instrumental experiment courses is mainly based on teacher evaluation. Students are passively accepted. Long-term success will inevitably lead to the lack of emotional acceptance of students. Therefore, aiming at the deficiency of the current evaluation system, colleges and universities should strengthen the reform of the assessment system for experimental courses, and transfer the initiative for evaluation to the students. The teachers will play a role in guiding and supervising the assessment. Pay attention to and encourage students' self-evaluation and mutual evaluation.

\section{Acknowledgement}

Fund Project: Xi'an science and technology project

Project Number: 2017CGWL17

\section{References}

[1] Kong Ling, Jing Rong, Zhao Fengda, et al. Multi WSN mobile robot dynamic clustering data collection method [J]. Small Computer Systems, 2014, 35 (4): 845-849.

[2] Borrego C, Castillo S, Robles S. Striving for sensing: Taming your mobile code to share a robot sensor network [J]. Information Sciences, 2014, 277(2):338-357.

[3] Torres-González A, Dios M D, Ollero A. Range-only SLAM for robot-sensor network cooperation [J]. Autonomous Robots, 2017(4):1-15.

[4] Sakurama K. Multi-robot Formation Control over Distance Sensor Network [J]. IFAC-PapersOnLine, 2016, 49(22):198-203.

[5] Chun J, Kim S, Lee S, et al. A Hazardous Substance Monitoring Sensor Network Using Multiple Robot Vehicle [J]. 2015, 15(1):147-155.

[6] Song B, Lu X, Bai X. Zigbee Based Wireless Sensor and Actuator Network for Service Robot Intelligent Space [J]. Wireless Sensor Network, 2012, 4(10):235-242.

[7] Li Jian, Li Shengjin, Lu Gang, et al. Based mobile robot wireless sensor networks [J] micro motor, 2013, 41 (8): 26-29.

[8] Zhao Peng, Sun Mei, Qian Haizhong, et al. Mobile Robot wireless sensor network data collection method [J]. Electronics, 2013, 36 (6): 881-884. 
[9] Axenie C, Conradt J. Cortically inspired sensor fusion network for mobile robot egomotion estimation [J]. Robotics \& Autonomous Systems, 2015, 71:69-82. 